\title{
ABDOMINAL GUNSHOT INJURIES ADMITTED TO THE ROYAL CITY OF DUBLIN HOSPITAL DURING THE REBELLION.
}

\author{
By R. ATKINSON STONEY, F.R.C.S.I.;
}

Visiting Surgeon to the Roral City of Dublin Hospital.

[Read hefore the Section of Surgery, Tamuar, 19, 1917.]

WHEN asked by the Secretary if I had any subject on which I could read a short paper this evening, I thought that it might be of interest to the members of the profession here to-night if I were to give a brief account of the cases of abdominal gunshot wounds that were treated at the Royal City of Dublin Hospital during the rebellion last Easter. The number of cases is so small, and the conditions found so varied that I am afraid that no very definite conclusions can be drawn from them, except that, perhaps, the first case emphasises the fact that no case should be looked upon as absolutely hopeless. From the experience gained in the present European war our ideas have undergone a complete change from those that were formed after the Boer War. The general experience then was that the best chance of recovery after abdominal gunshot wounds was treatment by rest, starvation and morphia, whereas operation was almost invariably fatal, and seemed to remove any chance of recovery that the patient might have had. As I say the experience of the present war is the exact opposite of this, the best chance afforded of the patient's recovery is immediate operation. This apparent contradiction of observed facts is probably due to several 
causes. In the South African War most of the wounds were caused by rifle fire at long ranges, transport was difficult and delayed, operations could only be performed in adverse circumstances and often unsuitable surroundings. The soldiers in many instances had not had food for a considerable time before being wounded, and often the stomach and intestines must have been more or less empty. In the present war all these circumstances are changed, most of the wounds are produced by shell fragments or rifle bullets fired at close range, transport arrangements are much better, in many cases the wounded are brought to the casualty clearing stations within two hours of being hit; and within a few miles of the firing line there are hospitals and surgeons fully equipped and capable of undertaking abdominal operations under the most favourable conditions. Finally, as the fighting has become more or less stationary: commissariat arrangements are almost perfect and the soldier well fed, so that the alimentary canal is not likely to be empty. In some places the French have built special dug-outs close to or in the firing trenches where abdominal operations may be undertaken, and so the patient operated on without delay. Here in Dublin during the rebellion the conditions were practically ideal for the treaiment of the wounded, as in most cases they could be brought to a thoroughly equipped hospital and operated on by competent surgeons, as a rulc, within an hour or so of being wounded; and certainly in the case of general gunshot wounds the results were extremely satisfactory, and very little suppuration of a serious character was seen, in spite of the fact that many of the injuries were caused by ricochet or deformed bullets, fired at close range.

Curious to say, all the cases of abdominal wounds at the 
Royal City of Dublin Hospital occurred among civilians. The following is a brief description of them :-

Here I wish to express my indebtedness to my colleague, Mr. Johnston, who has kindly allowed me to include the cases under his charge.

W. R. was admitted to hospital on Tuesday, April 25th, 1916, about 10.30 a.m. He had been shot about half an hour previously close to the railway bridge on South Lott's Roar, and was brought to the hospital by the Pembroke Ambulance. There was a medium-sized round of entry directly in the middle line at the level of the second lumbar spine, and a large ragged wound of exit, big enough to admit two fingers just below the right costal margin in the nipple line. There was also a large superficial lacerated wound of the right forearm, immediately below the bend of the elbow, evidenily caused by the same bullet. The patient was brought from the ambulance into the special dispensary, which had been converted for the time being into a receiving and dressing room. As soon as his wounds were superficially examined, he was given a hypodermic of morphia, and immediately brought up to the operating theatre, and anæsthetised with ether. While the anæsthetic was being administered the anterior abdominal wound was washed with Fusol, and then the surrounding slin was painted with iodine. The mans condition was bad, the pulse was scarcely perceptible, and respirations were rapid and shallow; he was very pale, and the right side of the abdomen was dull on percussion. There was continuous oozing of blood from the abdominal wound, and from the condition of the man's clothing the hæmorrhage must have been very extensive. The wound was enlarged by a vertical incision of about four inches, and as soon as the abdomen was opened fresh hæmorrhage of an alarming character occurred; this was partially controlled by thrusting the left hand upwards against the liver, where a large irregular cavity was ielt. With the right hand large masses of clotted blood were removed from the right kidney pouch and flank, 
and the intestines were packed off with gauze swabs. It was then seen that there was a large hole, nearly the size of the closed fist, involving the anterior surface and sharp margin of the right lobe of the liver, with fissures extending from it in various directions; several pieces of liver up to a couple of inches in size were almost completely detached; indeed, it seemed as if almost the half of the right lobe had been burst and pulped so much so that it appeared quite hopeless to attempt closure with sutures. Iodoform gauze was therefore packed between the outer and anterior surface of the liver and the diaphragm and abdominal wall, and also under the liver all round the injured area, and then the cavity in the liver was packed tightly with iodoform gauze. By this means the hæmorrhage was checked, except for a very slight oozing. On one of the torn fragments of liver a vein as thick as a quill tooth-pick was exposed for nearly two inches, with most of its tributory branches torn. The patient's condition was so bad during the operation that a subcutaneous saline injection was started before the operation was completed, and over $1 \frac{1}{2}$ pints were injected under the skin of the left axilla and chest. The abdominal wall was closed with throughand-through silk-worm gut sutures and Nichel's clips. After the operation, which took less than half-an-hour, the patient was left on the theatre table surrounded by hot-water bottles and with the head lowered for an hour before he was sent back to the ward, where the foot of the bed was raised. The following day the dressings, which were soaked through with blood and bile, were changed. Except for severe pain, which necessitated the use of morphia for the first four days, and the onset of a considerable degree of jaundice accompanied by free escape of bile from the wound, the patient did well, and the plugs were gradually withdrawn, and the last of them was finally removed on Thursday, May 4th, and the stitches were taken out on the 6 th. The bowels were not moved till the fifth day, when castor oil was given; at no time did the temperature rise above $100^{\circ}$. The jaundice persisted for some time and the wound closed slowly, a large piece of liver substance coming away as a slough about ten days after the

T. 
operation. The patient finally left the hospital completely healed on June 12th, seven weeks after his admission. When I saw him shortly before last Christmas he appeared in normal health, except for a ventral hernia in the region of the scar, and a complaint of general weakness. $\mathrm{He}$ is now back at work. From the appearance of the wound in the back, which was evidently the wound of entrance, it is probable that it was caused by a ricochet bullet, and this explains the extensive injury done to the liver. The shot was probably fired from the direction of Beggar's Bush Barracks, at a range of between 200 and 300 yards.

CASE 2.-Bridget $\mathrm{s}$., aged 13, was admitted at 5 p.m. on Friday afternoon, April 28th, 1916. She had been shot about one hour previously, and it was reported that she had vomited some blood. There was a small wound in the anterior abdominal wall about two inches above and to the right of the umbilicus, and another wound in the left lumbar region behind. Her condition was serious, blanched, fast pulse and respiration and some dulness in either flank, with distension of the abdomen. A vertical median incision was made, and on opening the abdominal cavity it was found full of partially clotted blood, and fresh hæmorrhage continued freely. The stomach was first examined, and a wound was found involving both the greater curvature and the great omentum; this wound did not penetrate to the interior of the stomach, and was only bleeding slightly. Then a double perforation of the small intestine was found, also a perforation of the mesentery close to its root, from which the chief hæmorrhage appeared to be proceeding. There was also a tear of the descending colon not involving the lumen and a perforation of the descending mesocolon. All the perforations were closed and the hæmorrhage checked, most of the clots and fluid blood were cleared out, and the abdomen filled with hot saline fluid and the abdominal wall closed with through-and-through silkworm gut sutures and Michel's clips. Saline was also injected into the axilla during the concluding steps of the operation, which lasted forty minutes. When returned to the ward the 
patient's condition was not too bad, but she died suddenly about midnight. Here we were unable to find out at what range the shot had been fired, but the wounds were probably produced by a modern service rifle.

CASE 3.-A. B., a man of 46 , was admitted to hospital cn Saturday afternoon in a very collapsed condition. There were two abdominal wounds, a large one in the right nipple line one inch under the margin of the ribs, with everted omentum. A smaller wound was present in the left lateral wall of the abdomen. Mr. Johnston opened the abdomen through the right wound but found no serious damage in the neighbourhood; at the same I opened the abdomen through the left linea semilunaris and found an extensive perforation of the descending colon, which I closed; hæmorrhage was still proceeding from nearer the middle line, and it was then found that the small intestine close to the duodeno-jejunal flexure was extensively torn: it was completely divided about ten inches from the flexure, and completely divided again about 18 inches lower down, and between the two divisions there was an irregular longitudinal tear of nearly three inches in length. The portion between the complete divisions was resected and an end-to-end anastomosis effected; the mesentery tied off, the blood clots removed and the abdominal cavity filled with hot saline, and the incisions closed with throughand-through silk-worm gut sutures. The patient never rallied, and died about two hours after the operation. The injuries in this case were probably caused by either a ricochet or a blunt-nosed bullet, or else one of the large, soft-leaden sporting bullets so extensively used by the rebels, as the smaller of the two wounds, that in the left lateral abdominal wall, was considerably larger than would be caused by a modern bullet.

There were two other cases which may be included, though neither of them was operated on at the hospital.

CASE 4.-This was an old man who was brought in by the Pembroke Ambulance on Sunday-he had been shot through 
the abdomen from side to side about an hour previously. The wounds were both small, and situated the one in the right and the other the left lateral abdominal wall. When admitted his condition was so bad that it was not thought advisable to operate, and he died a few hours afterwards, without recovering from the condition of shock in which he was when first seen.

CASE 5 was also an elderly man, who was admitted late one night. $\mathrm{He}$ had been shot in the right inguinal region, and there were no symptoms to determine the track of the missile which had lodged. No definite abdominal symptoms had developed by the following day, when the was removed from the hospital and operated on, I believe, elsewhere. We never heard what was found at the operation, and the only information given was that the patient died.

Though the general result of these cases was so disappointing, all except the first proving fatal, yet it must be admitted that they tend to confirm the teaching of the present day, that early operation is the only hope in cases of abdominal gunshot injuries. The first case, the rupture of the liver, was an unexpected success, for the condition of the patient before operation seemed almost hopeless, and even after the conclusion of the operation his chances of recovery appeared to be extremely small. Here operation, fortunately performed early, was undoubtedly lifesaving. In the second case, the young girl with multiple injuries to stomach, small and large intestine, there stemed to be a chance of recovery after the operation, in spite of the severity of the injuries. Probably, if the patient had been older or more robust, or if the operation could have been done half an hour earlier, success migh $_{t}$ have crowned our efforts. In the third case, the man with complete double division of the small intestine, and 
with injury to the large bowel also-the condition was probably hopeless, owing to the extensive nature of the injuries, and all that can be said is that he could not have lived more than an hour or two in any case. In the fourth case, that of the elderly man, his age and condition on admission precluded any hope of a successful operation, and in the last case, while the patient was under our charge, there were no definite indications of abdominal trouble, and when the question of exploratory operation was suggested it was at once refused. One lesson was deeply impressed upon me by these cases, namely, that in abdominal gunshot injuries hæmorrhage is of cardinal importance, and all one's efforts should be at once directed to its immediate control. While the abdomen is closed there is a positive intra-abdominal pressure which increases pari passu with the bleeding, and therefore to some extent controls and checks the hæmorrhage; as soon, however, as the abdominal wall is incised this pressure disappears, and blecding, which up till then has gradually lessened, and may perhaps have ceased, starts again with redoubled fury, and may prove most alarming. It is therefore most necessary for the operator, when he has discovered one injury to the viscera, not to waste time in repairing it before he has made certain that there are no other and more important injuries, and when he finds that the injuries are multiple that which is giving rise to the greatest hæmorrhage should be the first to receive attention. In some cases, where serious multiple injuries exist, time may be saved if the assistant of a second operator undertakes the repair of some of the lesions whilst the surgeon himself is repairing the others, and case 3 may even occur where, as in the third case reported, two abdominal operations through separate incisions may with 
advantage be carried out at one and the same time. In this work, above all others, everything must be done to minimise the duration and shock of the operation, and also to try and counteract the shock from which the patient is already suffering, partly as the result of the injury and partly as the result of the consequent hæmorphage. 